\title{
2D molecular precursor for a one-pot synthesis of semiconducting metal sulphide nanocrystals
}

\author{
ABHIJIT BERA and BHAGAVATULA L V PRASAD* \\ Physical/Materials Chemistry Division, National Chemical Laboratory (CSIR-NCL), Pune 411008, India \\ *Author for correspondence (pl.bhagavatula@ncl.res.in)
}

MS received 19 March 2018; accepted 1 May 2018; published online 6 September 2018

\begin{abstract}
D molecular materials, namely, metal alkyl thiolates, have been used as a single-source precursor for the synthesis of semiconducting metal sulphide nanocrystals (NCs) by thermal decomposition. These 2D molecular precursors have all the ingredients required for metal sulphide synthesis (metal source, sulphur source and protecting ligand). In this study, we demonstrate a simple and general 'solvothermal decomposition' approach for the synthesis of high-quality $\mathrm{Cu}_{2} \mathrm{~S}$, $\mathrm{PbS}, \mathrm{CdS}, \mathrm{MnS}$ and $\mathrm{ZnS}$ NCs. The size of the NC can also be controlled by changing the decomposition temperature. Furthermore, the optical properties of the NCs have also been studied.
\end{abstract}

Keywords. Single-source precursor; nanocrystal; thermal decomposition; optical properties.

\section{Introduction}

Over the past few decades, there has been great interest in the utilization of semiconducting nanocrystals (NCs) for biomedical [1-3], electronic [4-6] and alternative-energy applications [7-9]. This interest stems from their optical properties, which can be tuned by particle size via quantum confinement or changing the composition. Even a slight change of size, morphology or composition of NCs has a considerable effect on the optical properties, including the energies of the absorption onset, fluorescence emission and surface plasmons [10]. Thus, the synthesis of monodisperse, single-crystalline metal sulphide NCs is highly desired. While there are many ways to synthesize NCs, solution-based colloidal synthesis often yields the high-quality materials, with desired monodispersity displaying uniform properties [10]. Over the past several years, several groups have reported the synthesis of metal sulphide NCs using various synthesis routes, including the thermolysis of single-source precursors [11-13], solvent-less synthesis [14], thermolysis of metal-oleylamine complexes [15] and a simple organic-amine-assisted hydrothermal process [16].

In this communication, a general 'solvothermal decomposition' approach has been carried out for synthesizing metal sulphide NCs. It is a direct-heating approach without any subsequent reagent injection. Both the 'directheating' and 'hot-injection' processes are very convenient and are suitable for large-scale synthesis. However, to obtain high-quality NCs, the hot-injection method poses some drawbacks as the reagents used are characterized with high reactivity [17]. Therefore, modulating size via this 'hotinjection' process becomes tricky for many systems. On the other hand, the 2D molecular precursors (metal alkyl thiolates) that we propose here have ideal reactivity and have all the ingredients required for metal sulphide synthesis (metal source, sulphur source and protecting ligand). Because of their low reactivity below a certain temperature (i.e., the reaction temperature) a short nucleation process can be achieved simply by direct heating of these metal thiolates at or above the decomposition temperature. Thus, at these high temperatures, the decomposition of thiols leads to the formation of metal sulphides and at the termination of the reaction, the particles get in-situ capped with a layer of intact thiols. Furthermore, metal thiolates of different metals are easy to prepare in large scale by a simple solvent-less process, which are highly stable in air below the decomposition temperature. Accordingly, we show in this work that by the decomposition of a suitable metal thiolate in a high boiling solvent like 1-octadecene a series of metal sulphide $\mathrm{NCs}$, such as $\mathrm{Cu}_{2} \mathrm{~S}, \mathrm{PbS}, \mathrm{ZnS}, \mathrm{MnS}$ and $\mathrm{CdS}$, can be successfully synthesized. Interestingly their size also can be controlled by varying the decomposition temperature. This method avoids injecting a second reagent during the reaction and uses air-free manipulation, which is very suitable for large-scale synthesis. 


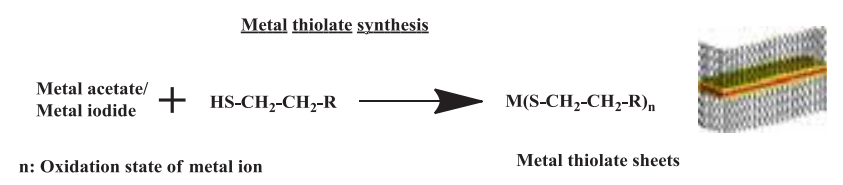

Scheme 1. Schematic representation of the metal thiolate synthesis.

\section{Experimental}

\subsection{General procedure for metal sulphide NCs}

A quantity of $200 \mathrm{mg}$ of metal thiolates powder was taken along with $15 \mathrm{ml}$ of 1 -ODE in a $100 \mathrm{ml} \mathrm{RB}$ flask. First, the reaction mixture was heated at mild temperature (130$140^{\circ} \mathrm{C}$ ); the homogeneous clear solution formed was then further heated at high temperature $\left(200-240^{\circ} \mathrm{C}\right)$ for $30 \mathrm{~min}$. This reaction mixture was precipitated and washed with methanol to obtain pure metal sulphide NCs. These products were re-dispersed into toluene or tetrachloroethylene and characterized by PXRD and UV-vis absorption, fluorescence spectra and transmission electron microscopy (TEM).

\section{Results and discussion}

A series of 2D molecular precursors (metal thiolates of different metals like $\mathrm{Cu}, \mathrm{Pb}, \mathrm{Cd}, \mathrm{Mn}$ and $\mathrm{Zn}$ ) were synthesized following our previously reported protocol [18].

Briefly, metal precursors (such as metal acetate or metal iodide) were taken in a glass vial and alkyl thiol (octane thiol) was added into this (the generic procedure for metal thiolate synthesis is shown in scheme 1). This immediately led to a colour change due to $\mathrm{M}-\mathrm{S}$ co-ordination (synthesis and cleaning of materials are explained in detail in the Experimental section in supplementary information). These metal alkyl thiolates are coordination compounds and exist as lamellae or stacked sheets in the solid state [18]. The PXRD patterns of different metal thiolates are shown in figure 1a, which shows periodically spaced $(00 l)$ reflections. SEM images (shown in figure $1 b-f$ ) of these metal thiolates also clearly indicate that all of the metal thiolates are featured with layer-like structures and justify the sheet-like (2D) morphology. It is confirmed by energy-dispersive X-ray analysis (see supplementary figure S1) that the stoichiometry of metal thiolates is $\mathrm{M}_{n}(\mathrm{SR})_{n}$ (where $\mathrm{M}=\mathrm{Cu}, \mathrm{Pb}, \mathrm{Cd}, \mathrm{Mn}$ and $\mathrm{Zn}$ and $n=$ oxidation state of metal). This simple and generic solvent-less synthesis of $2 \mathrm{D}$ single-source precursors is scalable and the resulting materials are also highly stable in air [18].

Subsequently, the semiconducting metal sulphide NCs were synthesized using these $2 \mathrm{D}$ molecular precursors as a starting material (the generic procedure for metal sulphide synthesis is shown in scheme 2). The main advantage is that the 2D metal thiolates used here are single-source precursors,
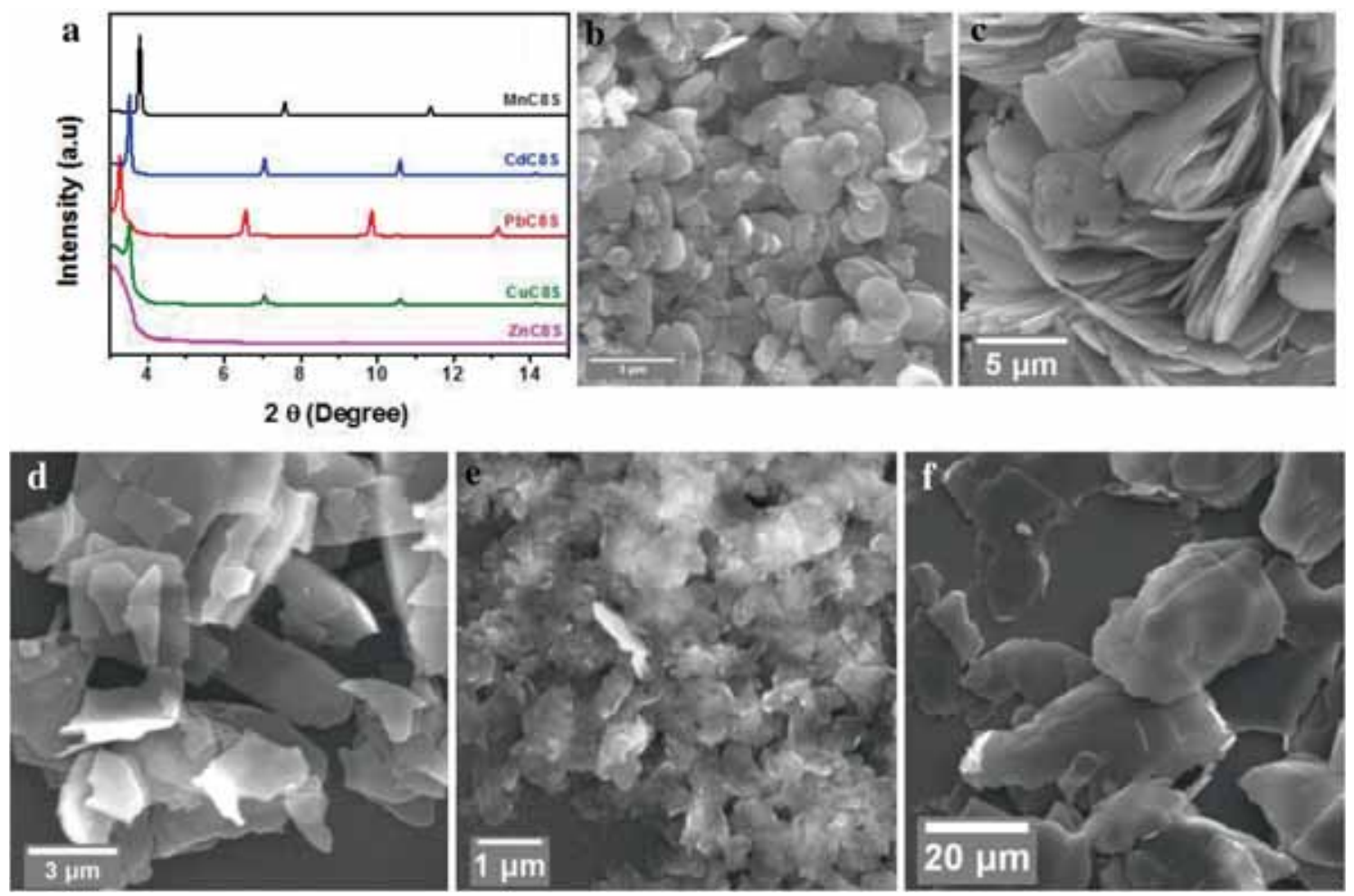

Figure 1. (a) PXRD of metal thiolates; PXRD of manganese octanethiolate $\left(\mathrm{MnC}_{8} \mathrm{~S}\right)$ is in black, cadmium octanethiolate $\left(\mathrm{CdC}_{8} \mathrm{~S}\right)$ in blue, lead octanethiolate $\left(\mathrm{PbC}_{8} \mathrm{~S}\right)$ in red, copper octanethiolate $\left(\mathrm{CuC}_{8} \mathrm{~S}\right)$ in green and zinc octanethiolate $\left(\mathrm{ZnC}_{8} \mathrm{~S}\right)$ in pink. $\mathrm{SEM}$ images of metal thiolates: (b) $\mathrm{CuC}_{8} \mathrm{~S}$, (c) $\mathrm{CdC}_{8} \mathrm{~S},(\mathbf{d}) \mathrm{PbC}_{8} \mathrm{~S}$, (e) $\mathrm{ZnC}_{8} \mathrm{~S}$ and (f) $\mathrm{MnC}_{8} \mathrm{~S}$. 


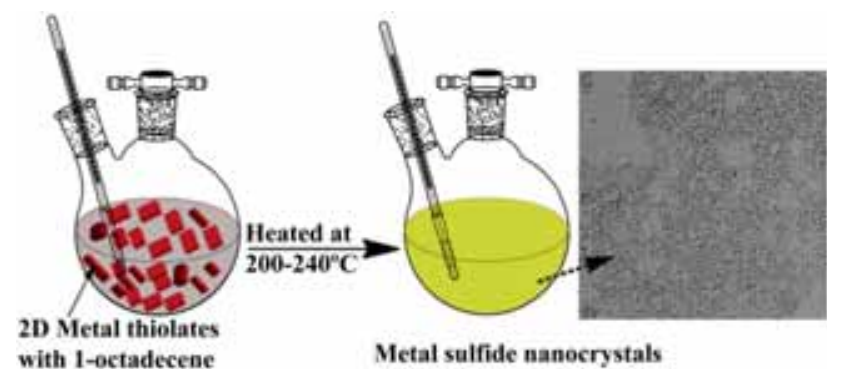

Scheme 2. Method for generic metal sulphide NCs synthesis.

\section{Thermal decomposition of metal thiolates}

$$
\underset{\mathrm{Cd}\left(\mathrm{S}-\mathrm{CH}_{2}-\mathrm{CH}_{2}-\mathrm{R}\right)_{2}}{\stackrel{\Delta}{240}{ }^{\circ} \mathrm{C}} \begin{gathered}
\mathrm{CdS} \\
+\mathrm{R}-{ }_{2} \mathrm{HC}-{ }_{2} \mathrm{HC}-\mathrm{H}-\mathrm{CH}_{2}-\mathrm{CH}_{2}-\mathrm{R}
\end{gathered}
$$

Scheme 3. Proposed reaction mechanism for thermal decomposition of metal thiolates to metal sulphide NCs (following Ref. [19]). The equation has not been balanced.

where all the ingredients required for metal sulphide NCs are inbuilt in their structure (metal source, sulphur source and capping agent). These metal thiolates are highly stable and are dispersible at mild temperatures $\left(120-140^{\circ} \mathrm{C}\right)$ in a high boiling solvent like 1-octadecene. After or at a certain temperature (decomposition temperature) the uniform dispersion of metal thiolates (which have been shown to exist as individual sheet-like structures in solution) [18] can decompose into metal sulphide and an alkene [19] and some of the undecomposed alkyl thiol passivate the surface of the NCs to control the growth (proposed reaction mechanism is shown in scheme 3, according to Choi et al [19]). In the following, the synthesis procedure is explained in detail through the synthesis of cadmium sulphide NCs as an example. First, $200 \mathrm{mg}$ of the Cd-octanethiolate 2D molecular complex was taken along with a solvent (1-octadecene) and the temperature was raised to $130^{\circ} \mathrm{C}$ to get a homogeneous clear solution. The temperature was then raised to $200-240^{\circ} \mathrm{C}$ in argon flow and the reaction was kept at this temperature for $30 \mathrm{~min}$. The colourless solution became pale yellow, indicating formation of CdS. The heating was stopped immediately after the colour change and the system was allowed to naturally cool to room temperature (which took $\sim 45 \mathrm{~min}$ ). The product of this reaction was collected by precipitating it with acetone/ethanol addition, and after washing it two times with acetone/ethanol (centrifuged at $5000 \mathrm{rpm}$ for $3 \mathrm{~min}$ ) the precipitate was dried in argon flow. The precipitate was re-dispersed into tetrachloroethylene or toluene for various measurements. The PXRD pattern of the purified materials clearly matched those of the CdS cubic phases (for PXRD pattern of these samples, see supplementary figure S2), which corresponds to JCPDS card No\#43-1469. Figure 2 shows the UV-vis absorption spectra of CdS NCs synthesized between 200 and

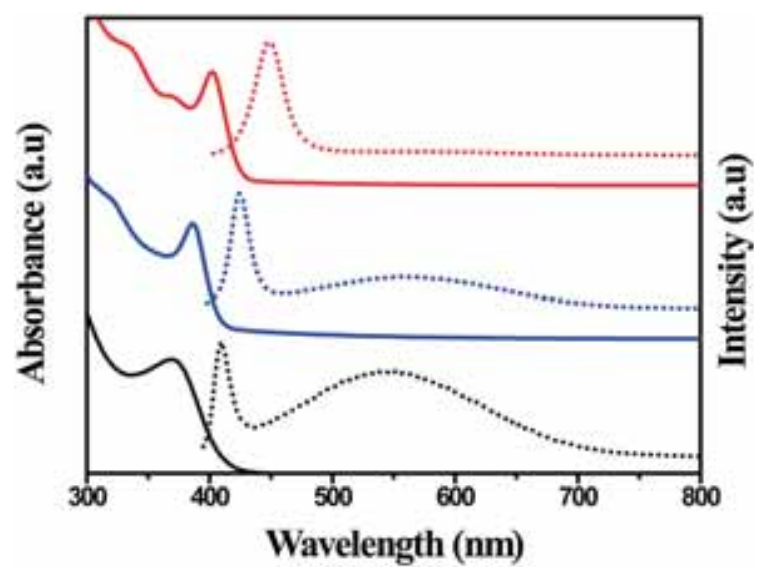

Figure 2. Absorption (solid line) and emission (dotted line) spectra of CdS NCs synthesized at different temperatures $\left(200-240^{\circ} \mathrm{C}\right)$.

$240^{\circ} \mathrm{C}$. As can be noticed, all spectra exhibit sharp bandgap absorption features, indicating the formation of nearly monodisperse CdS NCs. It may be noticed that the samples display a well-defined excitonic peak and the peak position varied gradually from 370 to 385 to $405 \mathrm{~nm}$ as the reaction temperature was varied to 200 to 220 to $240^{\circ} \mathrm{C}$ (figure 2). The absorption peak also becomes narrow and the peak shifts towards red (figure 2). The photoluminescence (PL) of CdS NCs (figure 2) shows both a sharp bandgap emission as well as trap state emission centred around $450-700 \mathrm{~nm}$. The trap state emission is significantly depressed on increasing reaction temperature and bandgap emission dominates the fluorescence of CdS NCs for synthesis temperatures over $200^{\circ} \mathrm{C}$ (figure 2).

TEM images show near-spherical NCs with average size of $\sim 3 \mathrm{~nm}$ and the size of CdS NCs can be tuned between $\sim 3$ and $5 \mathrm{~nm}$, with longer reaction times and higher reaction temperatures favouring the formation of larger NCs (figure $3 a-c$ ).

Similar to the synthesis of CdS NCs, nearly monodisperse sub-13 nm PbS NCs can be synthesized by heating Pboctanethiolate precursor in $\mathrm{ODE}$ solution at $200^{\circ} \mathrm{C}$. The PXRD pattern of the purified materials clearly matched that of the $\mathrm{PbS}$ with rock salt structure (see supplementary figure S2), which corresponds to JCPDS card No\#05-0592. TEM images showed NCs with nearly spherical shape and average size of $13 \mathrm{~nm}$ (figure 3d). As in the case of $\mathrm{CdS}$ and $\mathrm{PbS}$ NCs, monodisperse $\sim$ sub- $3 \mathrm{~nm} \mathrm{ZnS}$ and $\mathrm{MnS}$ can also be synthesized by thermal decomposition of zinc octanethiolate and $\mathrm{Mn}$-octanethiolate, respectively, in ODE at $240^{\circ} \mathrm{C}$. The PXRD pattern of the purified materials shows zinc blende ZnS phase (supplementary figure S2; JCPDF no-75-1546), and the average size of NCs in both these cases was found to be $\sim 3.5 \mathrm{~nm}$ (see figure $3 \mathrm{e}$ for TEM image of $\mathrm{ZnS} \mathrm{NCs}$ and figure $3 \mathrm{f}$ for TEM image of MnS NCs).

The PL of ZnS NCs (figure 4a) shows a sharp bandgap emission at $\sim 410 \mathrm{~nm}$. The PXRD pattern of MnS NCs (supplementary figure S2) is broad in nature, which we ascribe 

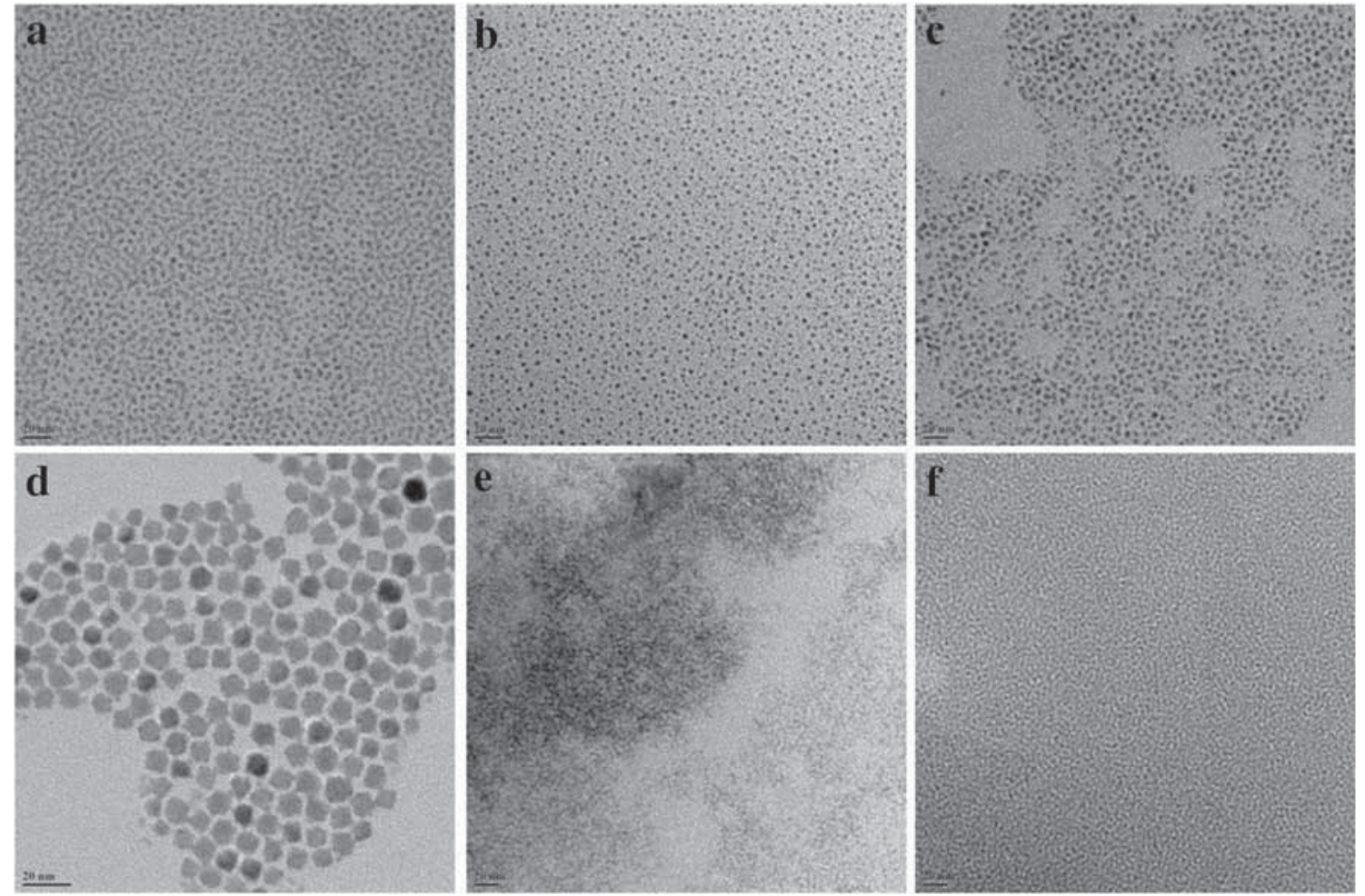

Figure 3. TEM images of different metal sulphide quantum dots synthesized using their corresponding thiolates as precursors. CdS at different temperatures: (a) 200, (b) 220 and (c) $240^{\circ} \mathrm{C}$. (d) $\mathrm{PbS}$ at $200^{\circ} \mathrm{C}$, (e) $\mathrm{ZnS}$ at $200^{\circ} \mathrm{C}$ and (f) $\mathrm{MnS}$ at $200^{\circ} \mathrm{C}$. In all images, the scale bar corresponds to $20 \mathrm{~nm}$.
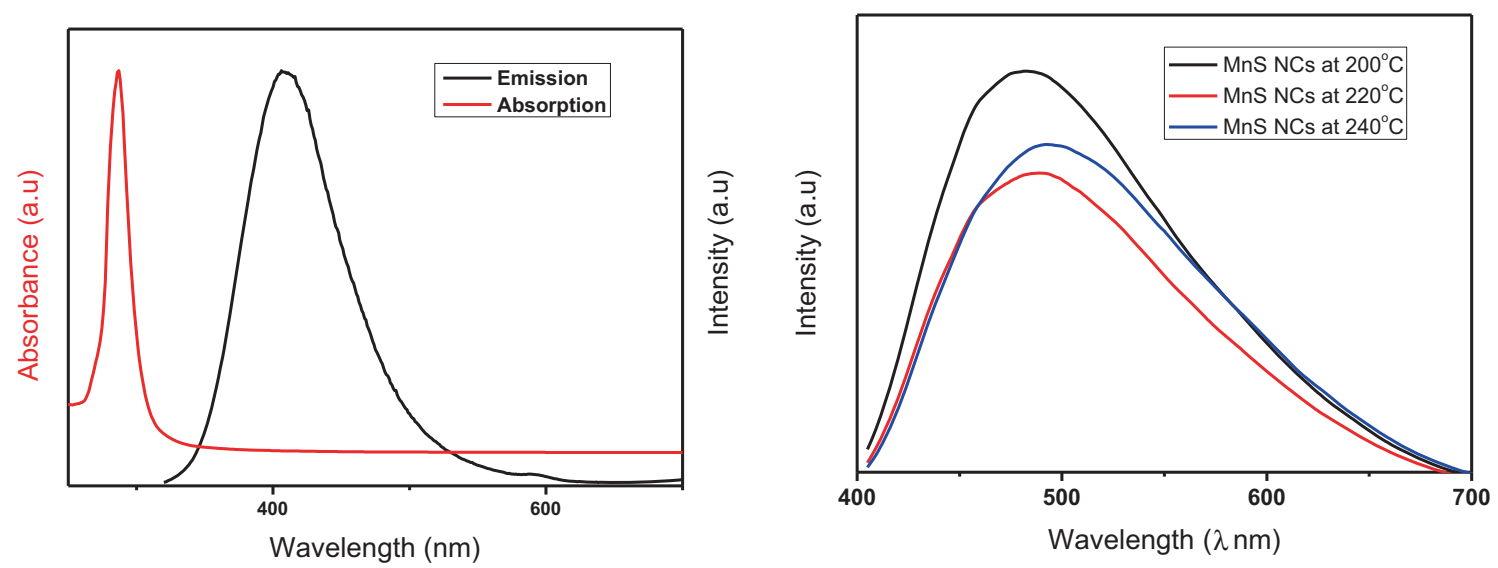

Figure 4. Optical spectra of $\mathrm{ZnS}$ and $\mathrm{MnS}$ NCs. (a) Absorption (red line) and emission (black line) spectra of $\mathrm{ZnS}$ NCs. (b) Emission spectra of MnS NCs synthesized at different temperatures.

to the small-sized particles $(2.5-3 \mathrm{~nm})$. The PL of MnS NCs, synthesized at different reaction temperatures, shows a sharp bandgap emission range of 470-490 $\mathrm{nm}$ (figure $4 \mathrm{~b}$ ). The TEM image of the MnS NCs synthesized at $200^{\circ} \mathrm{C}$ (figure $3 \mathrm{f}$ ) reveals the presence of small spherical-shaped NCs.

Similar to the synthesis of different NCs described earlier, monodisperse $\mathrm{NCs}$ of $\sim 6-10 \mathrm{~nm} \mathrm{Cu}_{2} \mathrm{~S}$ were also synthesized by the thermal decomposition of copper octanethiolate in $\mathrm{ODE}$ at $200-240^{\circ} \mathrm{C}$. For this, first, $200 \mathrm{mg}$ of the $\mathrm{Cu}$-octanethiolate (prepared using cupper acetate as $\mathrm{Cu}$ source) 2D molecular complex was taken in a solvent (1-octadecene) and the temperature was raised to $130^{\circ} \mathrm{C}$ to get a homogeneous clear solution. The temperature was then raised to $200^{\circ} \mathrm{C}$ in argon flow and the reaction was kept at this 

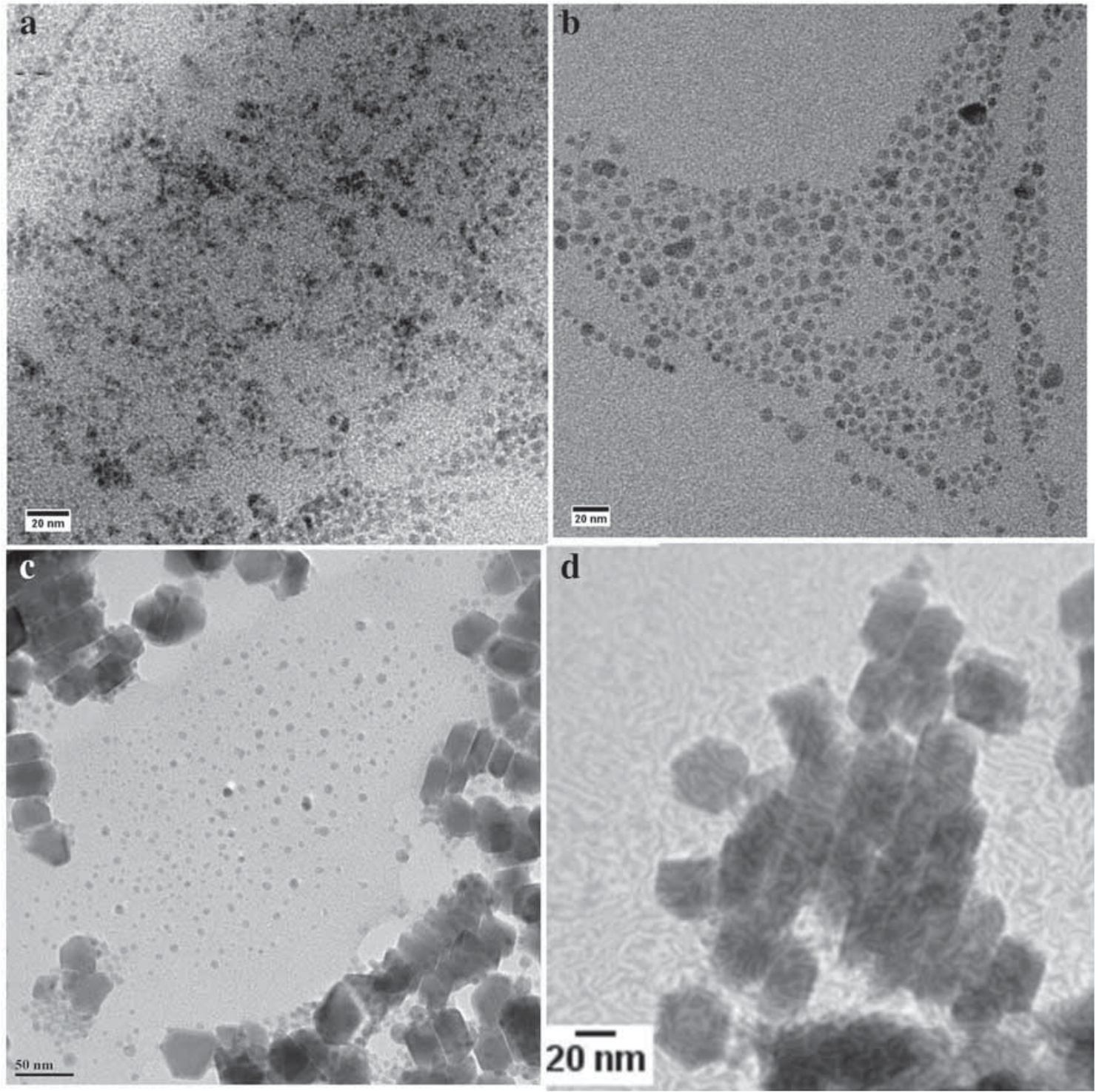

Figure 5. TEM images of $\mathrm{Cu}_{2} \mathrm{~S}$ prepared by thermolysis from $\mathrm{Cu}$-octanethiolate $\left(\mathrm{Cu}(\mathrm{ac})_{2}\right.$ as metal precursor) at (a) 200 and (b) $240^{\circ} \mathrm{C}$. (c, d) Effect of iodide in thermolysis of $\mathrm{Cu}$-octanethiolate (CuI as metal precursor); mixed shape of particles formed (like plates, sheets as well as spherical particles are present).

temperature for $30 \mathrm{~min}$. The pale yellow coloured solution became brownish black, indicating formation of $\mathrm{Cu}_{2} \mathrm{~S}$. The heating was stopped immediately after the colour change; the system was allowed to naturally cool to room temperature (which took $\sim 45 \mathrm{~min}$ ) and later the material was purified, similar to the CdS NCs synthesis. The PXRD pattern of the purified materials clearly matched with the chalcocite $\mathrm{Cu}_{2} \mathrm{~S}$ structure (for PXRD pattern of these samples, see supplementary figure S2), which corresponds to JCPDS 84-0206. TEM images (figure 5a) reveal that the NCs are almost uniform in size and spherical in shape with a particle diameter of $\sim 6 \mathrm{~nm}$. One of the notable features of this synthesis procedure is the tunability of NCs size by changing the reaction temperature. For instance, when the $\mathrm{Cu}$-thiolate clear solution (obtained by heating the $\mathrm{Cu}$-thiolate in 1-octadecene to $130^{\circ} \mathrm{C}$ ) was heated to $240^{\circ} \mathrm{C}$ for $30 \mathrm{~min}, \sim 10$-nm-sized spherical $\mathrm{Cu}_{2} \mathrm{~S}$ NCs were obtained (TEM images shown in figure $5 b$ ).

Interestingly, not only the size of $\mathrm{Cu}_{2} \mathrm{~S} \mathrm{NCs}$ but also the shape (morphology) can be controlled by changing the metal source during synthesis of metal thiolate preparation. For instance, we found that the high-temperature decomposition of $\mathrm{Cu}$-octanethiolate prepared from $\mathrm{CuI}$ at $240^{\circ} \mathrm{C}$ in 1-ODE resulted in the formation of plate-like $\mathrm{Cu}_{2} \mathrm{~S}$ NCs (with very few spherical NCs, see figure $5 \mathrm{c}$ and d). This is in agreement with the results of Wu et al [20], where they reported that introduction of halide ions slows down the growth kinetics and therefore the $\mathrm{Cu}_{2} \mathrm{~S}$ NCs with plate-like morphology 
are more favoured. In our case as the $\mathrm{Cu}$-octanethiolate was prepared from $\mathrm{CuI}$, some iodide ions were found to be left behind as confirmed from the EDS results (see supplementary figures S3 and S4) of the Cu-octanethiolate sample. We strongly believe that these iodide ions act as shape-directing agents, resulting in the formation of $\mathrm{Cu}_{2} \mathrm{~S}$ plates, similar to the previous studies [20].

We believe that the simple process described here can be easily extended to prepare heterostructure of different metal sulphides like $\mathrm{CdS}-\mathrm{ZnS}, \mathrm{CdS}-\mathrm{MnS}$ and $\mathrm{ZnS}-\mathrm{MnS}$; $\mathrm{Cd}_{x} \mathrm{Zn}_{1-x} \mathrm{~S}, \mathrm{Zn}_{x} \mathrm{Cd}_{1-x} \mathrm{~S}$, etc. by simply heating the corresponding metal thiolates (in a proper ratio) in a high boiling solvent. It is well known that the afore-mentioned heterostructure NCs show high quantum yield; hence these type of heterostructures are expected to be highly useful in LED (optoelectronic devices) applications. Similarly, synthesis of doped NCs like Mn:CdS and Mn:ZnS could also be attempted by heating manganese thiolates (taken in a proper ratio) with cadmium or zinc thiolates in a high boiling solvent.

\section{Conclusion}

In conclusion, a simple and general direct-heating solvothermal decomposition synthesis method using 2D molecular precursor (metal thiolates as single-source precursor) has been developed for the synthesis of different metal sulphide $\mathrm{NCs}$ like $\mathrm{Cu}_{2} \mathrm{~S}, \mathrm{PbS}, \mathrm{CdS}, \mathrm{MnS}$ and $\mathrm{ZnS}$. The synthesis of starting material (metal thiolate 2D sheets) is also very simple and scalable. We also showed that the size and shape of the NCs could be controlled by changing the reaction temperature and changing the metal precursor (used for metal thiolate preparation).

\section{Acknowledgements}

AB thanks UGC, New Delhi, for research fellowship. We thank the Council of Scientific and Industrial Research
(CSIR), New Delhi, for financial assistance through the 12th

Five-Year Plan Project (CSC 0134).

\section{References}

[1] Medintz I L, Uyeda H T, Goldman E R and Mattoussi H 2005 Nat. Mater. 4435

[2] Brunchez M P, Moronne M, Gin P, Weiss S and Alivisatos A P 1998 Science $\mathbf{2 8 1} 2013$

[3] Chan W C and Nie W S 1998 Science 2812016

[4] Talapin D V, Lee J S, Kovalenko M V and Shevchenko E V 2010 Chem. Rev. 110389

[5] Filankembo A, Giorgio S, Lisiecki I and Pileni M P 2003 J. Phys. Chem. B 1077492

[6] Pileni M P 1997 Langmuir 133266

[7] Chen X B, Li C, Gratzel M, Kostecki R and Mao S S 2012 Chem. Soc. Rev. 417909

[8] Sakamoto T, Sunamura H, Kawaura H, Hasegawa T, Nakayama T and Aonob M 2003 Appl. Phys. Lett. 823032

[9] Hu J, Li L S, Yang W, Manna L, Wang L W and Alivisatos A P 2001 Science 2922060

[10] Park J, Joo J, Kwon S G, Jang Y and Hyeon T 2007 Angew. Chem. Int. Ed. 464630

[11] Lee S M, Jun Y W, Cho S N and Cheon J 2002 J. Am. Chem. Soc. 12411244

[12] Lee S M, Cho S N and Cheon J 2003 Adv. Mater. 15441

[13] Aldana J, Lavelle N, Wang Y and Peng X 2005 J. Am. Chem. Soc. 1272496

[14] Chen L, Chen Y B and Wu L M 2004 J. Am. Chem. Soc. 126 16334

[15] Yu J H, Joo J, Park H M, Baik S I, Kim Y W, Kim S C et al 2005 J. Am. Chem. Soc. 1275662

[16] Lu Q, Gao F and Zhao D 2002 Nano Lett. 2725

[17] Murray C, Norris D J and Bawendi M G 1993 J. Am. Chem. Soc. 1158706

[18] Busupalli B, Kummara S, Kumaraswamy G and Prasad B L V 2015 Chem. Mater. 263436

[19] Choi S H, An K, Kim E G, Yu J H, Kim J H and Hyeon T 2009 Adv. Funct. Mater. 191645

[20] Wu W Y, Chakrabortty S, Chang C K L, Guchhait A, Lin M and Chan Y 2014 Chem. Mater. 266120 